\title{
AN ASYMPTOTICALLY NONEXPANSIVE COMMUTATIVE SEMIGROUP WITH NO FIXED POINTS
}

\author{
DARYL TINGLEY ${ }^{1}$
}

\begin{abstract}
An example of an asymptotically nonexpansive commutative semigroup with no fixed points is presented.
\end{abstract}

1. Introduction. Throughout this paper, $\mathscr{F}$ will represent a commutative semigroup (under composition) of self mappings of a weakly compact set $K$ in a real Banach space $X$. A fixed point for the semigroup $\mathscr{F}$ is a point $x \in K$ such that $f(x)=x$ for all $f \in \mathscr{F}$, denoted $\mathscr{F}(x)=x$. The existence of fixed points for commutative (and weaker) semigroups, having some type of nonexpansive condition, has been investigated by many authors (cf. $[1-11,14])$. We list some of these conditions.

Definitions. Let $K$ be a subset of a Banach space $X$, and let $\mathscr{F}=\left\{f_{\alpha}: \alpha \in A\right\}$ be a commutative semigroup of self mappings of $K$. Then $\mathscr{F}$ is said to be eventually nonexpansive if

(a) for each $f_{\alpha} \in \mathscr{F}$ there is a real number $k_{\alpha}$ such that $\left\|f_{\alpha}(x)-f_{\alpha}(y)\right\| \leqslant$ $k_{\alpha}\|x-y\|$ for all $x, y \in K$, and

(b) for every $\varepsilon>0$ there is a $\gamma \in A$ such that $k_{\beta}<1+\varepsilon$ whenever $f_{\beta} \in f_{\gamma} \mathscr{F}=$ $\left\{f_{\gamma} f: f \in \mathscr{F}\right\}$.

If all of the constants $k_{\alpha}$ in (a) are one, then $\mathscr{F}$ is said to be nonexpansive.

The semigroup $\mathscr{F}$ is said to be ultimately nonexpansive if each $f \in \mathscr{F}$ is continuous and for every $x, y \in K$ and for every $\varepsilon>0$ there is an $f \in \mathscr{F}$ such that for all $g \in \mathscr{F}$,

$$
\|f g(x)-f g(y)\| \leqslant(1+\varepsilon)\|x-y\| .
$$

If this is satisfied with $\varepsilon=0$ then $\mathscr{F}$ is said to be asymptotically nonexpansive.

In the case that $\mathscr{F}$ is nonexpansive or eventually nonexpansive it has been shown for a wide variety of Banach spaces $X$ (most notably uniformly convex and those with normal structure) that $\mathscr{F}$ always has a fixed point (cf. $[3,7,14])$.

On the other hand, for $\mathscr{F}$ an asymptotically or the more general ultimately nonexpansive semigroup, some additional conditions have always been used. Such

Received by the editors June 20, 1984 and, in revised form, April 8, 1985.

1980 Mathematics Subject Classification. Primary 47H10; Secondary 46A35, 46A40.

Key words and phrases. Fixed point, nonexpansive, cone, semigroup.

${ }^{1}$ The author would like to thank Professors M. Edelstein and J. Borwein for many useful discussions and suggestions. 
conditions include the assumption that $K$ be compact (cf. [8]) or a condition on the orbits of points under $\mathscr{F}$ (cf. $[\mathbf{5}, \mathbf{8}]$ ).

The question as to whether or not there is necessarily a fixed point for $\mathscr{F}$, a commutative asymptotically (or ultimately) nonexpansive semigroup, has not been addressed in the above mentioned papers; however it would seem to be the natural result to strive for. In this paper we present an example of an asymptotically nonexpansive semigroup (indeed, something much stronger) generated by a single function acting on a weakly compact subset of the Hilbert space $l^{2}$, which has no fixed points.

One of the reasons for studying asymptotically and ultimately nonexpansive semigroups is that the functions need not be Lipschitzian, or even uniformly continuous. Our example, coupled with the above mentioned results, suggests that a condition to insure some type of uniformity is necessary to guarantee the existence of fixed points of semigroups acting on weakly compact sets, even in a Hilbert space.

2. Preliminaries. In this section the definitions and preliminary results necessary for our example are presented. This consists of a brief introduction to cones associated with biorthogonal systems. These are considered in detail in [13].

Throughout this section $X$ will be a Banach space, $X^{*}$ its dual.

DEFINITIONS. Let

$$
\delta_{i j}=\left\{\begin{array}{ll}
1, & i=j, \\
0, & i \neq j,
\end{array} \quad \text { (i.e., the Kronecker } \delta\right) .
$$

If $\left\{x_{i}\right\} \subseteq X,\left\{x_{i}^{*}\right\} \subseteq X^{*}$ then $\left\{x_{i}, x_{i}^{*}\right\}$ is called a biorthogonal system for $X$ provided that $x_{i}^{*}\left(x_{j}\right)=\delta_{i j}$. The cone associated with a biorthogonal system, denoted $\mathscr{K}\left\{x_{i}, x_{i}^{*}\right\}$ is defined by

$$
\mathscr{K}\left\{x_{i}, x_{i}^{*}\right\}=\left\{x \in X \mid x_{i}^{*}(x) \geqslant 0\right\} .
$$

Biorthogonal systems are fundamental in the study of bases of Banach spaces (cf. [12]). For our purposes, we require only that $\left\{x_{i}\right\}$ be a "basis" for $\mathscr{K}\left(x_{i}, x_{i}^{*}\right)$. Indeed, we impose other conditions on the sequence $\left\{x_{i}\right\}$ that insure it is not a basis for $X$. (The condition $\lim _{n}\left(x_{n}, x_{n+1}\right)=1$ of Lemma 4 insures that the expansion operators associated with $\left\{x_{i}, x_{i}^{*}\right\}$ are not bounded. This contradicts [12, Chapter III, Corollary 2.3].)

DEFINITION. The sequence $\left\{x_{i}\right\}$ is said to be a basis for $\mathscr{K}\left\{x_{i}, x_{i}^{*}\right\}$ provided that for each $x \in \mathscr{K}\left\{x_{i}, x_{i}^{*}\right\}, x=\sum_{i=1}^{\infty} \lambda_{i} x_{i}$, for some sequence of scalers $\lambda_{i}$.

It follows immediately that $\lambda_{i}=x_{i}^{*}(x) \geqslant 0$.

Definition. A cone $\mathscr{K}$ is said to be normal if there exists a constant $\delta>0$ such that $\|x+y\|>\delta$ for $x, y \in \mathscr{K},\|x\|=\|y\|=1$. The biorthogonal sequence $\left\{x_{i}, x_{i}^{*}\right\}$ is said to be normal if $\mathscr{K}\left\{x_{i}, x_{i}^{*}\right\}$ is normal.

Conditions sufficient for $\left\{x_{i}\right\}$ to be a basis for $\mathscr{K}\left\{x_{i}, x_{i}^{*}\right\}$ are contained in the following result [13, Theorem 2].

THEOREM 1. Let $X$ be a Banach space, $\left\{x_{i}, x_{i}^{*}\right\}$ a biorthogonal system in $X$ such that the associated cone $\mathscr{K}\left\{x_{i}, x_{i}^{*}\right\}$ is normal and sequentially weakly complete. Then $\left\{x_{i}\right\}$ is a basis for $\mathscr{K}\left\{x_{i}, x_{i}^{*}\right\}$. 
Definition. A sequence $\left\{x_{i}\right\}$ in a Hilbert space $\mathscr{H}$ is said to be close if $\left(x_{i}, x_{j}\right) \geqslant 0$ for all $i$ and $j$. A biorthogonal sequence $\left\{x_{i}, x_{i}^{*}\right\}$ for $\mathscr{H}$ is said to be close if $\left\{x_{i}\right\}$ is close.

In $\$ 3$ a biorthogonal system which is both normal and close is used to construct the example. We then present such a system.

It is easy to verify that if $\left\{x_{i}, x_{i}^{*}\right\}$ is normal, then $\left\{x_{i}^{*}\right\}$ must be total over $X$. (That is, if $x_{i}^{*}(x)=0$ for all $i$, then $x=0$.) In a manner similar to the proof of Theorem 1 , it can be shown that if $\left\{x_{i}, x_{i}^{*}\right\}$ is a close biorthogonal system for a Hilbert space $\mathscr{H}$ and $\left\{x_{i}^{*}\right\}$ is total over $\mathscr{H}$, then $\left\{x_{i}\right\}$ is a basis for $\mathscr{K}\left\{x_{i}, x_{i}^{*}\right\}$ and then that $\left\{x_{i}, x_{i}^{*}\right\}$ is normal. Thus the assumption that $\left\{x_{i}, x_{i}^{*}\right\}$ is both normal and close can be replaced by the (formally) weaker assumptions that $\left\{x_{i}\right\}$ is close and $\left\{x_{i}^{*}\right\}$ is total over $X$.

Lemma 1. Let $\left\{x_{i}, x_{i}^{*}\right\}$ be a biorthogonal sequence in a Hilbert space $\mathscr{H}$ which is both close and normal such that $\left\|x_{i}\right\|=1$ for all $i$. If $x \in \mathscr{K}\left\{x_{i}, x_{i}^{*}\right\}$, then $\Sigma\left(x_{i}^{*}(x)\right)^{2}$ $\leqslant\|x\|^{2}$.

Proof. Theorem 1 shows that $x=\sum_{i=1}^{\infty} x_{i}^{*}(x) x_{i}$. Thus

$$
\|x\|^{2}=\sum_{i=1}^{\infty} \sum_{j=1}^{\infty} x_{i}^{*}(x) x_{j}^{*}(x)\left(x_{i}, x_{j}\right) \geqslant \sum_{i=1}^{\infty} x_{i}^{*}(x)^{2},
$$

since $\left(x_{i}, x_{j}\right) \geqslant 0$ and $\left\|x_{i}\right\|=1$ for all $i$ and $j$.

3. In this section we present the example of an asymptotically nonexpansive map, acting on a closed bounded subset of the Hilbert space $l^{2}$, which has no fixed point. We first do this in an abstract fashion, as this makes clear exactly what properties are needed, and then give a concrete version.

Let $\left\{x_{i}, x_{i}^{*}\right\}$ be a biorthogonal sequence for a Hilbert space $\mathscr{H}$, which is both normal and close, such that $\left\|x_{i}\right\|=1$. Theorem 1 then shows that for each $x \in$ $\mathscr{K}\left\{x_{i}, x_{i}^{*}\right\}, x=\sum x_{i}^{*}(x) x_{i}$.

Letting $B$ be the unit ball in $\mathscr{H}$, define $K=\mathscr{K}\left\{x_{i}, x_{i}^{*}\right\} \cap B$. This is the set that the semigroup will act upon.

For $x \in K$, let $g(x)=(1-\|x\|) x_{1}+\sum_{i=1}^{\infty} x_{i}^{*}(x)^{2} x_{i+1}$ and define the function $f$ by

$$
f(x)=\frac{g(x)}{\|g(x)\|} .
$$

Recalling Lemma 1, and noting that $g(x) \neq 0$, it is easy to verify that both $f$ and $g$ are well defined, and that $f(x) \in K$.

LEMMA 2. The function $f$, and hence the semigroup $\left\{f^{n}(x): n=1,2, \ldots\right\}$, has no fixed points.

Proof. This follows by noting that $f \mid f(K)$ is, loosely speaking, a shift on the basis $\left\{x_{i}\right\}$. 
LEMMA 3. The functions $f$ and $g$, defined above, are continuous on $K$.

Proof. Since the norm is continuous, it is sufficient to show that

$$
h(x)=\sum_{i=1}^{\infty} x_{i}^{*}(x)^{2} x_{i+1}
$$

is continuous on $K$. In fact we show that $h$ is continuous on $\mathscr{K}\left\{x_{i}, x_{i}^{*}\right\}$.

Let $y \in \mathscr{K}\left\{x_{i}, x_{i}^{*}\right\}$ be fixed, and assume that $\left\{y_{n}\right\} \subset \mathscr{K}\left\{x_{i}, x_{i}^{*}\right\}$ converges to $y$. Let $\varepsilon>0$ be given.

Since $y=\sum_{i=1}^{\infty} x_{i}^{*}(y) x_{i}, m$ can be chosen so that $\left\|\sum_{i=m+1}^{\infty} x_{i}(y) x_{i}\right\|<\varepsilon$. Since $x_{i}^{*}$, is continuous, $i=1,2, \ldots N$ can be chosen so that for $n>N$,

$$
\left\|\sum_{i=1}^{m} x_{i}^{*}\left(y_{n}\right) x_{i}-\sum_{i=1}^{m} x_{i}^{*}(y) x_{i}\right\|<\varepsilon \text { and }\left\|y_{n}-y\right\|<\varepsilon .
$$

Thus for $n>N$,

$$
\begin{aligned}
& \left\|\sum_{i=m+1}^{\infty} x_{i}^{*}\left(y_{n}\right) x_{i}\right\| \\
& \leqslant\left\|y_{n}-y\right\|+\left\|\sum_{i=1}^{m} x_{i}^{*}(y) x_{i}-\sum_{i=1}^{m} x_{i}^{*}\left(y_{n}\right) x_{i}\right\|+\left\|\sum_{i=m+1}^{\infty} x_{i}^{*}(y) x_{i}\right\| \\
& <3 \varepsilon
\end{aligned}
$$

and since $\left\{x_{i}\right\}$ is close and $\left\|x_{i}\right\|=1$ for all $i$,

$$
\begin{aligned}
\sum_{i=m+1}^{\infty} x_{i}^{*}\left(y_{n}\right)^{2} & \leqslant \sum_{i=m+1}^{\infty} \sum_{j=m+1}^{\infty} x_{i}^{*}\left(y_{n}\right) x_{j}^{*}\left(y_{n}\right)\left(x_{i}, x_{j}\right) \\
& =\left\|\sum_{i=m+1}^{\infty} x_{i}^{*}\left(y_{n}\right) x_{i}\right\|^{2} \leqslant 9 \varepsilon^{2} .
\end{aligned}
$$

Similarly, $\sum_{i=m+1}^{\infty} x_{i}^{*}(y)^{2} \leqslant \varepsilon^{2}$. For $n>N$,

$$
\begin{aligned}
\left\|h(y)-h\left(y_{n}\right)\right\| & =\left\|\sum_{i=1}^{\infty} x_{i}^{*}(y)^{2} x_{i+1}-\sum_{i=1}^{\infty} x_{i}^{*}\left(y_{n}\right)^{2} x_{i+1}\right\| \\
\leqslant & \left\|\sum_{i=1}^{m}\left[x_{i}^{*}(y)^{2}-x_{i}^{*}\left(y_{n}\right)^{2}\right] x_{i+1}\right\| \\
& +\sum_{i=m+1}^{\infty} x_{i}^{*}(y)^{2}+\sum_{i=m+1}^{\infty} x_{i}^{*}\left(y_{n}\right)^{2} .
\end{aligned}
$$

Since $x_{i}^{*}\left(y_{n}\right) \rightarrow x_{i}^{*}(y)$ for each $i=1,2, \ldots$, it follows that

$$
\lim \sup _{n}\left\|h(y)-h\left(y_{n}\right)\right\|<10 \varepsilon^{2},
$$

and since $\varepsilon$ was arbitrary that $h\left(y_{n}\right) \rightarrow h(y)$.

Thus $h(x)$ is continuous on $\mathscr{K}\left\{x_{i}, x_{i}^{*}\right\}$, and hence $f$ and $g$ are continuous on $K$.

Lemma 4. Assume that $\lim _{n}\left(x_{n}, x_{n+i}\right)=1$ for each $i=1,2, \ldots$ Then

$$
\lim _{n}\left\|f^{n}(x)-f^{n}(y)\right\|=0 \quad \text { for all } x, y \in K \text {. }
$$


Proof. Let $x \in K$ be fixed. It is sufficient to show that

$$
\lim _{n \rightarrow \infty}\left\|f^{n}(x)-f^{n}\left(x_{1}\right)\right\|=\lim _{n \rightarrow \infty}\left\|f^{n}(x)-x_{n+1}\right\|=0 .
$$

Let $\lambda_{i}^{(0)}=x_{i}^{*}(x), i=1,2, \ldots$, and $\lambda_{i}^{(n)}=x_{i+n}^{*}\left(f^{n}(x)\right), n=1,2, \ldots, i=0,1, \ldots$ Noting that $\|f(x)\|=1$, it follows that $x_{1}^{*}\left(f^{2}(x)\right)=1-\|f(x)\|=0$ and then that $x_{i}^{*}\left(f^{n}(x)\right)=0$ for $i=1,2, \ldots, n-1$. Thus $f^{n}(x)=\sum_{i=0}^{\infty} \lambda_{i}^{(n)} x_{i+n}, n=1,2, \ldots$, and $x=\sum_{i=1}^{\infty} \lambda_{i}^{(0)} x_{i}$.

We note that for $n \geqslant 1, \sum_{i=0}^{\infty} \lambda_{i}^{(n)}<\infty$, and that for any $i, j$,

$$
\frac{\lambda_{i}^{(n)}}{\lambda_{j}^{(n)}}=\left(\frac{\lambda_{i}^{(n-1)}}{\lambda_{j}^{(n-1)}}\right)^{2}=\left(\frac{\lambda_{i}^{(1)}}{\lambda_{j}^{(1)}}\right)^{2^{n-1}} .
$$

Let $k$ be the largest number such that $\lambda_{k}^{(1)}=\max _{j}\left\{\lambda_{j}^{(1)}\right\}$, let $\alpha=\lambda_{k}^{(1)}$ and let $\beta=\max _{j>k}\left\{\lambda_{j}^{(1)}\right\}$. Then for $i>k$,

$$
\frac{\lambda_{i}^{(n)}}{\lambda_{k}^{(n)}}=\left(\frac{\lambda_{i}^{(1)}}{\lambda_{k}^{(1)}}\right)^{2^{n-1}} \leqslant\left(\frac{\beta}{\alpha}\right)^{2^{n-2}} \frac{\lambda_{i}^{(1)}}{\lambda_{k}^{(1)}} .
$$

Using arguments similar to those used in Lemma 3, it is easy to see that $\lambda_{k}^{(n)} \leqslant 1$ for all $n$. Hence for $n \geqslant 2$,

$$
\sum_{i=k+1}^{\infty} \lambda_{i}^{(n)} \leqslant \sum_{i=k+1}^{\infty} \frac{\lambda_{i}^{(n)}}{\lambda_{k}^{(n)}} \leqslant\left(\frac{\beta}{\alpha}\right)^{2^{n-2}} \sum_{i=k+1}^{\infty} \frac{\lambda_{i}^{(1)}}{\lambda_{k}^{(1)}} .
$$

Since $\beta / \alpha<1, \lim _{n} \sum_{i=k+1}^{\infty} \lambda_{i}^{(n)}=0$. From this and the assumption of the lemma,

$$
\begin{aligned}
1 & =\lim _{n}\left\|f^{(n)}(x)\right\|^{2}=\lim _{n}\left\|\sum_{i=0}^{k} \lambda_{i}^{(n)} x_{i+n}\right\|^{2} \\
& =\lim _{n} \sum_{i=0}^{k} \sum_{j=0}^{k} \lambda_{i}^{(n)} \lambda_{j}^{(n)}\left(x_{i+n}, x_{j+n}\right)=\lim _{n} \sum_{i=0}^{k} \sum_{j=0}^{k} \lambda_{i}^{(n)} \lambda_{j}^{(n)} \\
& =\lim _{n}\left(\sum_{i=0}^{k} \lambda_{i}^{(n)}\right)^{2} .
\end{aligned}
$$

Thus

$$
\begin{aligned}
\lim _{n}\left\|f^{n}(x)-x_{n+1}\right\|^{2} & =\lim _{n}\left[\left\|f^{n}(x)\right\|^{2}+\left\|x_{n+1}\right\|^{2}-2\left(f^{n}(x), x_{n+1}\right)\right] \\
& =\lim _{n}\left[2-2 \sum_{i=0}^{k} \lambda_{i}^{(n)}\left(x_{i+n}, x_{n}\right)\right]=0 .
\end{aligned}
$$

Lemmas 2, 3 and 4 show that if $\left\{x_{i}, x_{i}^{*}\right\}$ is a biorthogonal close, normal sequence, with $\left\|x_{i}\right\|=1$ for all $i$, and such that $\lim _{n \rightarrow \infty}\left(x_{n}, x_{n+i}\right)=1$ for all $i$, then $\left\{f^{n}(x)\right.$ : $n=1,2, \ldots\}$ is a commutative asymptotically nonexpansive semigroup of functions, with no fixed points. We now present such a biorthogonal sequence. 
Let

$$
\begin{aligned}
& x_{1}=(1,0,0,0, \ldots), \\
& x_{2}=\left(0, \frac{1}{\sqrt{2}}, \frac{1}{\sqrt{2}}, 0,0, \ldots\right), \\
& x_{3}=\left(0,0, \frac{1}{\sqrt{2}}, \frac{1}{\sqrt{2}}, 0,0, \ldots\right), \\
& x_{4}=\left(0,0,0, \frac{1}{\sqrt{3}}, \frac{1}{\sqrt{3}}, \frac{1}{\sqrt{3}}, 0,0, \ldots\right) .
\end{aligned}
$$

In general: Define $m_{k}=\sum_{i=1}^{k} i=k(k+1) / 2$. For an arbitrary $n$, write $n$ as $m_{k}+j, 1 \leqslant j \leqslant k+1$. Let $e_{i}$ be the $i$ th standard basis vector of $l^{2}$.

Then define

$$
x_{n}=\frac{1}{\sqrt{k+1}} \sum_{i=0}^{k} e_{m_{k}+j+i} .
$$

Letting $x_{1}^{*}=e_{1}, x_{2}^{*}=e_{2}$ and, in general, with $n=m_{k}+j, 1 \leqslant j \leqslant k+1$, define $x_{n}^{*}=\sum_{i=j-1}^{k}\left(e_{m_{i}+j}-e_{m_{i}+j-1}\right)$ (where $e_{m_{0}+j}=0$ for all $\left.j\right)$. It can be verified that $\left\{x_{i}, x_{i}^{*}\right\}$ form a biorthogonal sequence which is both close and normal, $\left\|x_{i}\right\|=1$ and $\lim _{n}\left(x_{n}, x_{n+i}\right)=1$ for all $i$. Thus work in this section can be applied to $\left\{x_{i}, x_{i}^{*}\right\}$, producing a concrete example of an asymptotically nonexpansive commutative semigroup, acting on a weakly compact set in $l^{2}$, which has no fixed point.

We would like to point out that $\left\{f^{n}(x): n=1,2, \ldots\right\}$ satisfies a condition much stronger than asymptotically nonexpansive, since

$$
\lim _{n \rightarrow \infty}\left\|f^{n}(x)-f^{n}(y)\right\|=0 \text { for all } x, y \in K .
$$

This implies that $f$ is asymptotically regular (i.e., $\lim _{n \rightarrow \infty}\left\|f^{n}(x)-f^{n+1}(x)\right\|=0$ ). Asymptotically regular functions have frequently been used in fixed point theory (cf. $[1,6])$, in particular for theorems that characterize the location of fixed points.

Finally, it should be noted that $f$ is neither uniformly continuous nor uniformly asymptotically regular (cf. [6]), leaving open some obvious questions.

\section{REFERENCES}

1. F. E. Browder and W. V. Petryshn, The solution by iteration of nonlinear functional equations in Banach spaces, Bull. Amer. Math. Soc. 72 (1966), 571-575.

2. R. E. De Mann, Common fixed points for commuting contraction mappings, Pacific J. Math. 13 (1963). 1139-1141.

3. M. Edelstein, On nonexpansive mappings of Banach spaces, Proc. Cambridge Philos. Soc. 60 (1964), 439-447.

4. Fixed point theorems in uniformly convex Banach spaces, Proc. Amer. Math. Soc. 44 (1974), $369-374$.

5. M. Edelstein and M. T. Kiang, On ultimately nonexpansive semigroups, Pacific J. Math. 101 (1982), 93-102.

6. M. Edelstein and R. C. O'Brien, Nonexpansive mappings, asymptotic regularity, and successive approximations, J. London Math. Soc. (2) 17 (1978), 547-554.

7. K. Goebel and W. A. Kirk, A fixed point theorem for asymptotically nonexpansive mappings, Proc. Amer. Math. Soc. 35 (1972), 171-174. 
8. R. D. Holmes and P. P. Narayanaswami, On asymptotically nonexpansive semigroups of mappings, Canad. Math. Bull. 13 (1970), 209-214.

9. M. T. Kiang, Fixed point theorems for certain classes of semigroups of mappings, Trans. Amer. Math. Soc. 189 (1974), 63-76.

10. T. C. Lim, A fixed point theorem for families of nonexpansive mappings, Pacific J. Math. 53 (1974), 487-493.

11. A A fixed point theorem for multivalued nonexpansive mappings in a uniformly convex Banach space, Bull. Amer. Math. Soc. 80 (1974), 1123-1125.

12. J. T. Marti, Introduction to the theory of basis, Springer-Verlag, Berlin, 1969.

13. C. W. McArthur, I. Singer and M. Levin, On the cones associated with bio-orthogonal systems and bases in Banach spaces, Canad. J. Math. 21 (1969), 1206-1217.

14. D. W. Tingley, The construction of a fixed point for certain families of mappings, Applicable Anal. (to appear).

Department of Mathematics, Dalhousie University, Halifax, Nova Scotia, Canada B3H 4H8

Current address: Department of Mathematics, University of New Brunswick, Fredericton, New Brunswick. Canada. 